\title{
X-Ray Phase-Contrast Imaging with Nanoradian Angular Resolution
}

\author{
P. C. Diemoz, ${ }^{1,2}$ M. Endrizzi, ${ }^{1}$ C. E. Zapata, ${ }^{1,2}$ Z. D. Pešić, ${ }^{3}$ C. Rau, ${ }^{3}$ A. Bravin, ${ }^{4}$ I. K. Robinson, ${ }^{2,5}$ and A. Olivo ${ }^{1,2}$ \\ ${ }^{1}$ Department of Medical Physics and Bioengineering, UCL, WC1E 6BT London, United Kingdom \\ ${ }^{2}$ Research Complex at Harwell, Harwell Oxford Campus, OX11 OFA Didcot, United Kingdom \\ ${ }^{3}$ Diamond Light Source, Harwell Oxford Campus, OX11 ODE Didcot, United Kingdom \\ ${ }^{4}$ European Synchrotron Radiation Facility, 38043 Grenoble, France \\ ${ }^{5}$ London Centre for Nanotechnology, WC1H OAH London, United Kingdom
}

(Received 16 November 2012; published 28 March 2013)

\begin{abstract}
We present a new quantitative $\mathrm{x}$-ray phase-contrast imaging method based on the edge illumination principle, which allows achieving unprecedented nanoradian sensitivity. The extremely high angular resolution is demonstrated theoretically and through experimental images obtained at two different synchrotron radiation facilities. The results, achieved at both very high and very low x-ray energies, show that this highly sensitive technique can be efficiently exploited over a very broad range of experimental conditions. This method can open the way to new, previously inaccessible scientific applications in various fields including biology, medicine and materials science.
\end{abstract}

X-ray phase-contrast imaging (XPCI) has been the subject of intensive research in recent years. The sensitivity to the phase shift produced by the imaged object (in addition to absorption) leads to greatly increased contrast. The improvement with respect to conventional absorptionbased methods is apparent, and is particularly important for materials made of light elements and when high x-ray energies are employed, since in this case the absorption contrast can be very small.

Several phase-sensitive techniques have been developed and applied, which include crystal interferometry [1], analyzer-based imaging [2], free-space propagation [3], grating interferometric [4] and noninterferometric methods $[5,6]$. In this Letter we propose and apply a new quantitative method, falling in this last category, which aims at pushing even further the limits of the sensitivity obtainable with XPCI. We show, both theoretically and experimentally, that this method enables achievement of unprecedented angular resolution, about 1 order of magnitude higher than values previously reported for other XPCI techniques.

Our method is based on the edge illumination (EI) principle, which was first proposed and applied with synchrotron radiation (SR) at the end of the 1990s [5], and was later demonstrated to provide strong phase signals also when used with incoherent $\mathrm{x}$-ray beams provided by conventional laboratory sources [6,7]. The required setup is simple, scalable, and relatively insensitive to mechanical and thermal instabilities.

The EI principle consists in narrowing down the x-ray beam illuminating the object and analyzing the transmitted beam with the use of an absorbing edge placed in front of the detector. This is schematized in Fig. 1(a), where a pair of slits is used to narrow down the beam in one direction $(y)$, and the detector edge is positioned so as to stop part of the beam, while the remaining beam illuminates a line of detector pixels. $z_{\mathrm{sa}}$ indicates the distance between the source and the slits aperture, $z_{\mathrm{ad}}$ the distance between the aperture and the detector edge, $y_{\text {src }}$ the source size along $y$ and $a$ the size of the aperture. If a refracting object is inserted into the beam at a distance $z_{\text {od }}$ upstream of the edge-detector combination, photons previously incident on the edge can be deviated onto the detector pixels (increasing the detected signal), or the opposite can happen (decreasing the detected signal), according to the direction of refraction. An image can then be obtained by scanning the object through the beam in the direction orthogonal to the edge: this will be characterized by bright and dark fringes around the object edges, in addition to the absorption signal visible in the bulk regions of the sample. When a spatially extended beam, such as that provided by conventional sources, is used, the object scanning can be avoided by using appropriate masks, so that the EI principle can be repeated over the entire field of view $[6,7]$.

Recently, a method enabling the quantitative separation of absorption and refraction has been proposed and successfully tested with both incoherent (x-ray tubes) and coherent (synchrotron) radiation [8]. The algorithm is based on the assumption that the beam distribution incident on the detector edge is the geometric projection of the presample aperture on the detector, i.e., is either square (for a point source) or approximately Gaussian (for an extended source). However, when highly coherent radiation and large propagation distances are employed, wave diffraction effects have also to be taken into account. An example is reported in Fig. 1(b), where the beam spatial distribution $S_{\text {ref }}$ (i.e., the number of photons per unit length in the $y$ direction) obtainable with $y_{\text {src }}=24 \mu \mathrm{m}, a=$ $20 \mu \mathrm{m}, z_{\mathrm{ad}}=10.3 \mathrm{~m}$, and an x-ray energy $E=85 \mathrm{keV}$ is calculated by means of Fresnel diffraction integrals [9]. 


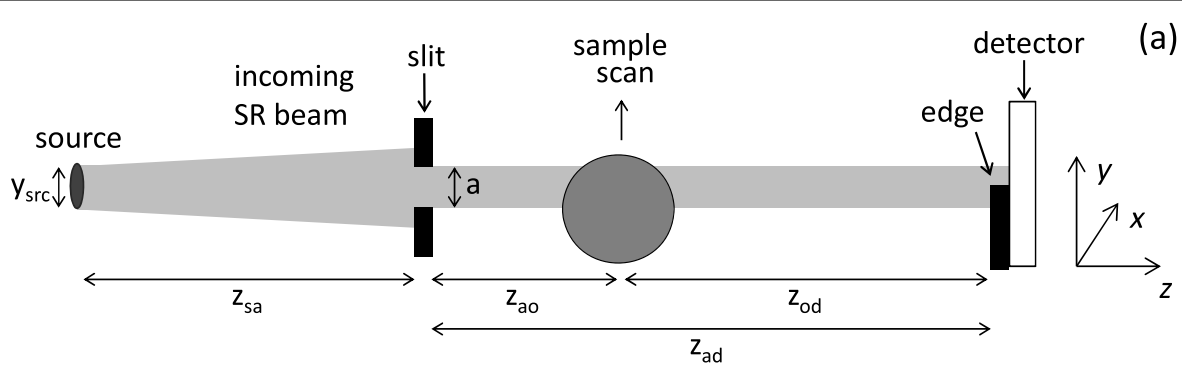

(a)
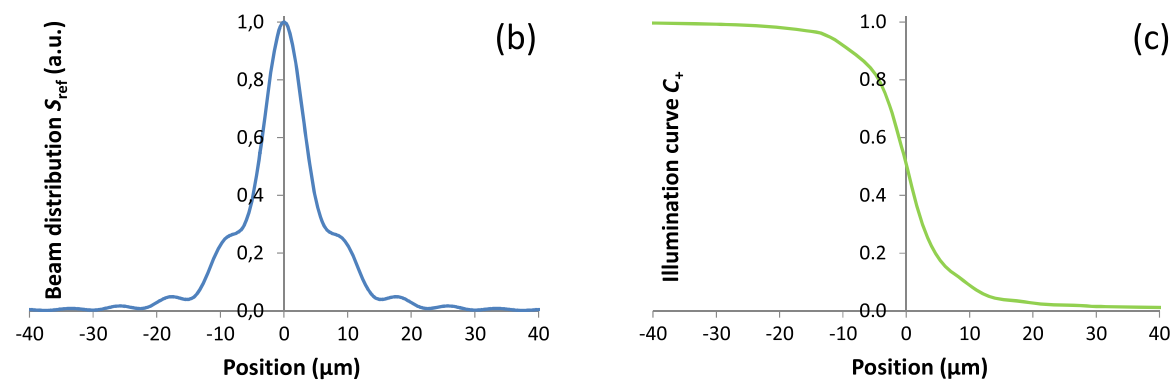

FIG. 1 (color online). (a) Scheme of the EI experimental setup (diagram is not to scale), (b) theoretical beam distribution for $y_{\text {src }}=$ $24 \mu \mathrm{m}, z_{\mathrm{sa}}=145 \mathrm{~m}, z_{\mathrm{ad}}=10.3 \mathrm{~m}$, and $E=85 \mathrm{keV}$ (parameters used for the experiment at the ESRF ID17 beam line), (c) corresponding illumination curve.

These parameters correspond to the experimental setup used at the ID17 beam line of the European Synchrotron Radiation Facility (ESRF, Grenoble, France). The beam distribution is in this case very different from that predictable by geometrical optics; therefore, a retrieval method based on this approximation would lead to errors in the calculation of the absorption and refraction information (see Supplemental Material [10]). We propose here a new method that can be adapted to the irregular beam shape provided by highly coherent beams and large propagation distances, and can therefore allow achieving extremely high angular sensitivity.

Let us consider the case of a monochromatic x-ray beam, a detector with efficiency $\varepsilon$, and let us discard the dependencies upon the coordinate $x$ parallel to the edge. When no sample is present in the beam, the number of photons recorded by the detector, for a certain position $y_{\text {edge }}$ of the edge along $y$, will be:

$$
I_{\text {ref },+}\left(y_{\text {edge }}\right)=\varepsilon \int_{y_{\text {edge }}}^{+\infty} d y S_{\text {ref }}(y)=C_{+}\left(y_{\text {edge }}\right) \varepsilon I_{0}
$$

where $I_{0}=\int_{-\infty}^{+\infty} d y S_{\text {ref }}(y)$ is the total number of photons in the beam and $C_{+}\left(y_{\text {edge }}\right) \equiv \int_{y_{\text {edge }}}^{+\infty} d y S_{\text {ref }}(y) / I_{0}$ is the illumination function [Fig. 1(c)], which is comprised between 0 and 1 . The + subscript indicates that the edge is chopping the lower part of the beam. $S_{\text {ref }}$ and $C_{+}$can be calculated by using Fresnel diffraction integrals [9]. In the general case, they depend in a complex way on several parameters, such as $E, y_{\text {src }}, z_{\text {sa }}, z_{\text {ad }}, a$, etc. Note that the illumination function $C_{+}$and its first derivative $S_{\text {ref }}$ can also be easily measured experimentally, by performing a scan of the detector edge along $y$.

When an object is inserted into the beam, two effects, absorption and refraction, can take place. As a consequence, for a certain position $p$ of the sample along $y$, the beam distribution incident on the detector slit is both reduced in amplitude and spatially shifted:

$$
S_{\text {obj }}(y ; p)=T(p) S_{\text {ref }}\left(y-z_{\text {od }} \cdot \Delta \theta_{y}(p)\right)
$$

where $T(p)$ is the object transmission and $\Delta \theta_{y}(p)$ is the component of the refraction angle along $y$. By combining Eqs. (1) and (2), and after the change of coordinates $y^{\prime}=$ $y-z_{\mathrm{od}} \Delta \theta_{y}(p)$, the following expression for the recorded intensity is obtained:

$$
\begin{aligned}
I_{\mathrm{obj},+}\left(y_{\text {edge }} ; p\right) & =\varepsilon \cdot T(p) \int_{y_{\text {edge }}-z_{\mathrm{od}} \Delta \theta_{y}(p)}^{+\infty} d y^{\prime} S_{\mathrm{ref}}\left(y^{\prime}\right) \\
& =T(p) \varepsilon I_{0} C_{+}\left(y_{\mathrm{edge}}-z_{\mathrm{od}} \Delta \theta_{y}(p)\right) .
\end{aligned}
$$

The effect of the object refraction therefore results in a change of the illumination level, equivalent to a shift of the position on the illumination curve [Fig. 1(c)].

Quantitative separation of the object absorption and refraction can be achieved by acquiring two complementary images, with the edge cutting either the lower ("plus" image) or the upper ("minus" image) half of the beam. Let us discard, for simplicity of notation, the dependency upon the object coordinate $p$, and indicate the intensities recorded by the detector in the two cases as $I_{\mathrm{obj},+}$ and $I_{\mathrm{obj},-}$, respectively. It is easy to verify that, in the latter case, the illumination function will be equal to 
$C_{-}\left(y_{\text {edge }}-z_{\text {od }} \Delta \theta_{y}\right)=1-C_{+}\left(y_{\text {edge }}-z_{\text {od }} \Delta \theta_{y}\right)$. We will also consider for simplicity that the required $50 \%$ illumination level is obtained with $y_{\text {edge }}=0$, i.e., $C_{-}(0)=$ $C_{+}(0)=0.5$. The calculation of the object transmission is straightforward, as the sum of $I_{\mathrm{obj},+}$ and $I_{\mathrm{obj},-}$ is equal to the intensity that would be measured without the detector edge. We can thus write

$$
T=\frac{I_{\mathrm{obj},+}+I_{\mathrm{obj},-}}{\varepsilon I_{0}} .
$$

Refraction, instead, can be calculated by subtracting the plus and minus images and making use of Eq. (4):

$$
\Delta \theta_{y}=-\frac{1}{z_{\mathrm{od}}} C_{+}^{-1}\left(\frac{I_{\mathrm{obj},+}-I_{\mathrm{obj},-}}{2\left(I_{\mathrm{obj},+}+I_{\mathrm{obj},-}\right)}+0.5\right) .
$$

Note that, $C_{+}$being a monotonic function, its inverse function is uniquely defined. The uncertainties in the retrieved transmission and refraction maps can be calculated by propagating the noise in Eqs. (4) and (5), respectively [for simplicity, we consider the same level of noise $\sigma\left(I_{\mathrm{obj}, \pm}\right)$ for the minus and plus images]:

$$
\begin{gathered}
\sigma(T)=\sqrt{2} \frac{\sigma\left(I_{\mathrm{obj}, \pm}\right)}{I_{0}} \simeq \frac{\sqrt{T}}{\sqrt{\varepsilon I_{0}}}, \\
\sigma\left(\Delta \theta_{y}\right)=\frac{1}{\sqrt{2} z_{\mathrm{od} d} S_{\mathrm{ref}, n}(0) T \varepsilon I_{0}} \sigma\left(I_{\mathrm{obj}, \pm}\right) \\
\simeq \frac{1}{2 z_{\mathrm{od}} S_{\mathrm{ref}, n}(0) \sqrt{T \varepsilon I_{0}}}
\end{gathered}
$$

where $S_{\text {ref, }, n} \equiv S_{\text {ref }} / I_{0}$ and where we have used the fact that $\left[d / d y\left(C_{+}^{-1}\right)\right](0)=1 / S_{\text {ref }, n}(0)$. The right-hand sides of Eqs. (6) and (7) have been obtained under the assumption of pure statistical (Poissonian) noise in the acquired images, i.e., $\sigma\left(I_{\mathrm{obj}, \pm}\right)=\sqrt{I_{\mathrm{obj}, \pm}}$.

Equation (7) represents a crucial result, since it enables the theoretical estimation of the achievable angular sensitivity. The amount of noise in the refraction angle image is in fact a measure of the system sensitivity, since it determines the magnitude of the angles that can be detected (i.e., the ones above the noise level). From Eq. (7) it can be seen that, besides being dependent on the number of detected photons, the angular resolution is determined by the quantity $z_{\mathrm{od}} S_{\mathrm{ref}, n}(0)$. This means that very high sensitivities will be obtained with a large propagation distance and a narrow beam profile at the detector plane. Note that the latter condition is equivalent to having a sharp illumination curve $C_{+}$(i.e., large $d C_{+} / d y$ ). It should be mentioned, however, that the two quantities are not independent and that the beam tends to broaden when the propagation distance is increased, as an effect of diffraction.

The method was experimentally demonstrated at two SR facilities, using different setups and acquisition conditions. The first test was carried out at the ID17 biomedical beam line of the ESRF. The source full width at half maximum
(FWHM) is about $132 \mu \mathrm{m}$ (horizontal) $\times 24 \mu \mathrm{m}$ (vertical), $z_{\mathrm{sa}}$ was $145 \mathrm{~m}, z_{\mathrm{ao}}=4.3 \mathrm{~m}, z_{\mathrm{od}}=6.0 \mathrm{~m}$, and $a=20 \mu \mathrm{m}$. Both the presample slits and the detector edge were oriented in the horizontal direction. A very high x-ray energy of $85 \mathrm{keV}$ was selected with a bent Laue-Laue $\mathrm{Si}(111)$ double-crystal monochromator, and the images were recorded with a $350 \mu \mathrm{m}$ pixel germanium detector [11]. Further details about the setup can be found in Ref. [12], in which unprocessed EI images, featuring mixed absorption and refraction information, were compared to those obtained with free-space propagation, showing high contrast improvement.

Minus and plus images were acquired, with an object scan step of $20 \mu \mathrm{m}$, for three filaments of different composition and dimensions: polyetheretherketone (PEEK) with diameters of $200 \mu \mathrm{m}$ and $450 \mu \mathrm{m}$, and aluminum with a diameter of $250 \mu \mathrm{m}\left(\delta_{\text {PEEK }}=3.79 \times 10^{-8}, \mu_{\text {PEEK }}=\right.$ $0.21 \mathrm{~cm}^{-1}, \delta_{\mathrm{Al}}=7.47 \times 10^{-8}$, and $\mu_{\mathrm{Al}}=0.52 \mathrm{~cm}^{-1}$ at $85 \mathrm{keV}$ [13]). Absorption and refraction images were calculated using Eqs. (4) and (5) and the experimentally measured illumination function [Figs. 2(a) and 2(b)], and intensity profiles across the three wires were compared with the theoretical ones [Figs. 2(c) and 2(d)]. The very good agreement with theory shows the high accuracy of the method, despite the fact that at this energy both the object absorption and refraction are very small (less than $1 \%$ and a fraction of $\mu \mathrm{rad}$, respectively). Furthermore, the noise in the refraction image (average of repeated calculations of the values standard deviation in 30 relatively large background regions of $40 \times 40$ pixels $^{2}$ ) was calculated to be $1.9 \pm 0.1 \mathrm{nrad}$. The obtained result is about 1 order of magnitude better than the value of 14 nrad previously published for grating interferometry, which was achieved at the ID19 beam line of the ESRF [14]. It is also much smaller compared to the angular resolution of about 65 nrad obtained with grating interferometry at the TOMCAT beam line of the Swiss Light Source [15]. Analyzer-based imaging was also shown to provide very high phase signals, but the value of about 15 nrad obtained in Ref. [16] indicates a sensitivity still 1 order of magnitude worse than the results presented in this work. This demonstrates the improved sensitivity of the proposed method, although we acknowledge that the result ultimately depends on the optical layout of the beam line used, particularly the distance from the source.

Each count on the germanium detector at $85 \mathrm{keV}$ corresponds to an average of 48 recorded photons [17], and about 30000 counts per pixel were collected for each of the plus and minus images. By using such photon numbers and the experimentally measured illumination function, a theoretical angular sensitivity of $2.1 \mathrm{nrad}$ can be predicted by Eq. (7), which is in very good agreement with the experimental value. This result suggests that in our setup the image noise is only due to statistical fluctuations and not to any structured noise (beam inhomogeneity, 


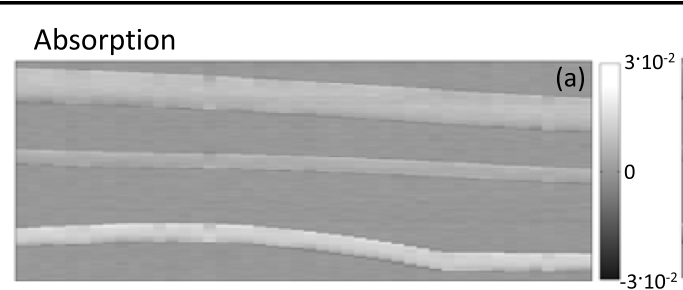

Refraction angle

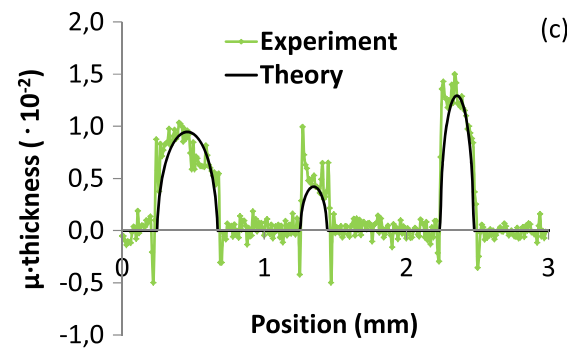

(c)

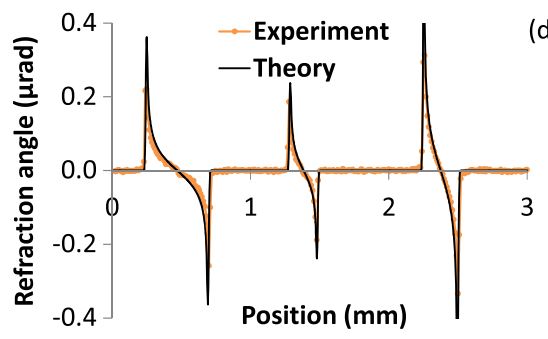

(d)

FIG. 2 (color online). (a) Absorption and (b) refraction images calculated for three filaments at $E=85 \mathrm{keV}$ : PEEK $450 \mu \mathrm{m}$ and $200 \mu \mathrm{m}, \mathrm{Al} 250 \mu \mathrm{m}$, from top to bottom. (c) Corresponding absorption and (d) refraction profiles across the wires and comparison with theory.

inhomogeneous detector response, roughness, or misalignment of the edge, etc.), indicating that further improvement in the sensitivity is possible by simply increasing the photon statistics. An additional gain in sensitivity in this setup may also be easily obtained by increasing the propagation distance. For example, the achievable distance $z_{\mathrm{od}}=10.3 \mathrm{~m}$ (corresponding to positioning the sample just downstream the slits) would lead to a potential improvement of about 1.7 times in the sensitivity [see Eq. (7)]. The object was in fact placed at a distance of $4.3 \mathrm{~m}$ from the slits only because of technical reasons (absence of a suitable sample stage at that position).

The second experiment was carried out at the ID13 beam line (Coherence branch) of the Diamond SR facility (Didcot, UK), in order to test the performance of the proposed method at very low $\mathrm{x}$-ray energies. The source size is about $400 \mu \mathrm{m}$ (horizontal) $\times 13 \mu \mathrm{m}($ vertical $)$, and the very large distance between the source and the presample slits, about $220 \mathrm{~m}$, provides an extremely coherent beam [18]. The beam line optics, such as the mirror and the Si(111) pseudochannel-cut crystal monochromator, are only horizontally deflecting, preserving the coherence in particular in the vertical direction. An x-ray energy of $12 \mathrm{keV}$ was used, $z_{\mathrm{ao}}$ was $9 \mathrm{~cm}, z_{\mathrm{od}}=14.69 \mathrm{~m}$, and $a=$ $20 \mu \mathrm{m}$. The images were acquired with the MEDIPIX detector, featuring a pixel size of $55 \mu \mathrm{m}$ [19].

A PEEK filament of diameter $165 \mu \mathrm{m}$, immersed in water, was imaged on the plus and minus positions by using a scan step of $5 \mu \mathrm{m}$. The refraction image, calculated by using the experimental illumination curve, is reported in Fig. 3(a): the refraction profile across the wire shows a very good agreement with the theory [Fig. 3(b)]. The noise in the refraction image was calculated to be $21 \mathrm{nrad}$, by considering a large background region of $20 \times 20$ pixels $^{2}$. The exposures gave about 39000 photons per pixel for each of the plus and minus images, providing a theoretical sensitivity of $13 \mathrm{nrad}$ [Eq. (7)]. The difference between the extracted and the theoretical values can be explained by the presence, in the calculated refraction image, of structured noise (weak linear artefacts) attributable to the nonhomogeneous detector response. This is confirmed by the fact that in smaller, artifact-free, $10 \times 10$ pixels $^{2}$ regions (number of regions $\sim 20$ ), the average calculated noise was only $15 \pm 3 \mathrm{nrad}$, thus in agreement with the theoretical predictions. A further improvement in the sensitivity of this setup would therefore require a precise calibration of the detector. A different angular resolution was obtained compared to the setup used at the ESRF ID17 beam line. This can be attributed to two main reasons: (a) the used photon statistics was larger in the image acquisition at the ESRF, and (b) at lower photon energies, diffraction leads to a larger broadening of the beam and therefore to a slight decrease in the sensitivity, according to Eq. (7).

(a)

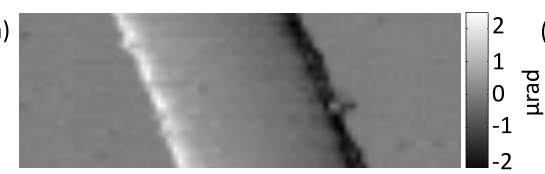

(b)
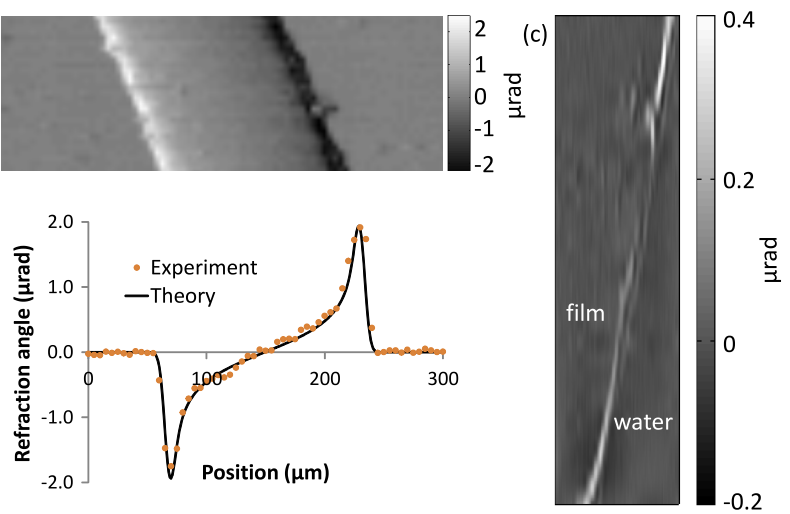

FIG. 3 (color online). (a) Refraction image of a PEEK filament (diameter $165 \mu \mathrm{m}$ ) in water, at $E=12 \mathrm{keV}$, (b) comparison between calculated and theoretical profiles across the wire, (c) refraction image of the edge of a $10 \mu \mathrm{m}$ thick polypropylene film in water. 
In order to demonstrate the extremely small signals detectable with this setup, an even more challenging sample was imaged: a $10 \mu \mathrm{m}$ thick polypropylene film in water, which has an almost matching refractive index $\left(\delta_{\mathrm{pol}}=1.41 \times\right.$ $10^{-6}$ and $\delta_{\text {wat }}=1.60 \times 10^{-6}$ at $12 \mathrm{keV} \mathrm{[20]).} \mathrm{A} 1 \mu \mathrm{m} \mathrm{scan}$ step was used in this case. Despite the very weak signal provided by this sample, the edge of the film is clearly visualized in the calculated refraction image [Fig. 3(c)]. We would like to point out that the faint signal provided by this object, as well as its dimensions, are similar to those one can expect from a single cell in a liquid environment. The obtained result, therefore, opens the way to new applications of $x$ rays in the imaging of single cells in biological samples.

It is important to underline that the very high angular resolution achievable with this method corresponds to very fine variations detectable in both the sample electron $\left(\rho_{e}\right)$ and mass density $(\rho)$. The refractive index decrement of a material, in fact, can be expressed as $\delta=\left(r_{0} \lambda^{2} / 2 \pi\right) \rho_{e} \simeq$ $\left(r_{0} \lambda^{2} / 4 \pi u\right) \rho=K(\lambda) \rho$, where $r_{0}$ is the classical electron radius and $u$ is the unified atomic mass unit [21]. It can be shown that $\Delta \theta_{y}(\lambda)=\partial\left(\int d z \delta(\lambda)\right) / \partial y=K(\lambda) \partial\left(\int d z \rho\right) / \partial y$ [22]; therefore, for a given angular resolution, the corresponding sensitivity to the object mass density variation is equal to $\sigma\left(\partial\left(\int d z \rho\right) / \partial y\right)=\sigma\left(\Delta \theta_{y}\right) / K$. For instance, for an object of constant thickness of $1 \mathrm{~cm}$ and at an energy of $12 \mathrm{keV}$, a $13 \mathrm{nrad}$ angular resolution leads to a detectable density gradient of about $9 \times 10^{-4} \mathrm{~g} / \mathrm{cm}^{3} \mathrm{~mm}^{-1}$ (e.g., a change of less than a thousandth of the density of water over a $\mathrm{mm}$ ). If one instead considers a high- $Z$ object with homogeneous density at $85 \mathrm{keV}$, this would enable detecting thickness gradients of a few $\mu \mathrm{m}$ over distances of the order of a tenth of a mm. With a $1 \mathrm{~nm}$ angular resolution, these sensitivities are increased 10-fold. This unparalleled level of sensitivity has the potential to revolutionize several fields, as, for example, material inspection, where ultrahigh precision in the produced components is often crucial [23].

We would like to stress that the high quantitative accuracy of this method is a direct result of taking into account the actual shape of the beam in the calculation of the absorption and refraction information. It can in fact be shown that a method neglecting the wave diffraction effects would provide incorrect results when highly coherent beams are considered (a demonstration of this is provided in the Supplemental Material [10]). The proposed quantitative method has been shown to be applicable in a variety of different experimental conditions. Not only were the experiments performed at different SR facilities, but they were also carried out by using two different detector technologies and employing both very high and very low x-ray energies, showing the great flexibility of the method. In particular, the very broad range of energies that can be efficiently used makes this technique suitable for the imaging of samples of various size and composition, in several fields including biology, medicine, cultural heritage, materials science, etc. The high quantitative accuracy of the method and the demonstrated unprecedented angular sensitivity open the way to imaging samples with a greatly increased level of detail, and will also enable new, previously inaccessible applications.

The authors would like to thank all personnel at beam lines I13 at Diamond and ID17 at the ESRF. This work was supported by the UK Engineering and Physical Sciences Research Council (Grants No. EP/G004250/1, No. EP/ I022562/1 and No. EP/I021884/1).

[1] U. Bonse and M. Hart, Appl. Phys. Lett. 6, 155 (1965).

[2] T. Davis, D. Gao, T. Gureyev, A. Stevenson, and S. Wilkins, Nature (London) 373, 595 (1995).

[3] A. Snigirev, I. Snigireva, V. Kohn, S. Kuznetsov, and I. Schelokov, Rev. Sci. Instrum. 66, 5486 (1995).

[4] C. David, B. Nöhammer, H. Solak, and E. Ziegler, Appl. Phys. Lett. 81, 3287 (2002).

[5] A. Olivo et al., Med. Phys. 28, 1610 (2001).

[6] A. Olivo and R. Speller, Appl. Phys. Lett. 91, 074106 (2007).

[7] P. R. T. Munro, K. Ignatyev, R. D. Speller, and A. Olivo, Opt. Express 18, 19681 (2010).

[8] P. R. T. Munro, K. Ignatyev, R. D. Speller, and A. Olivo, Proc. Natl. Acad. Sci. U.S.A. 109, 13922 (2012).

[9] P. R. T. Munro, K. Ignatyev, R. D. Speller, and A. Olivo, Opt. Express 18, 4103 (2010).

[10] See Supplemental Material at http://link.aps.org/ supplemental/10.1103/PhysRevLett.110.138105 for a demonstration of the improved quantitative accuracy of the proposed method compared to a method neglecting the diffraction effects.

[11] A. Bravin, S. Fiedler, P. Coan, J.-C. Labiche, C. Ponchut, A. Peterzol, and W. Thomlinson, Nucl. Instrum. Methods Phys. Res., Sect. A 510, 35 (2003).

[12] A. Olivo, P. C. Diemoz, and A. Bravin, Opt. Lett. 37, 915 (2012).

[13] R. J. Dejus and M. Sanchez del Rio, Rev. Sci. Instrum. 67, 3356 (1996).

[14] F. Pfeiffer, O. Bunk, C. David, M. Bech, G. Le Duc, A. Bravin, and P. Cloetens, Phys. Med. Biol. 52, 6923 (2007).

[15] P. Modregger, B. R. Pinzer, T. Thuring, S. Rutishauser, C. David, and M. Stampanoni, Opt. Express 19, 18324 (2011).

[16] P. C. Diemoz, A. Bravin, M. Langer, and P. Coan, Opt. Express 20, 27670 (2012).

[17] P. Coan, Ph.D. thesis, Université Joseph Fourier (Grenoble), 2006.

[18] C. Rau, U. Wagner, Z. Pešić, and A. De Fanis, Phys. Status Solidi A 208, 2522 (2011).

[19] C. Ponchut, J. Clément, J. M. Rigal, E. Papillon, J. Vallerga, D. LaMarra, and B. Mikulec, Nucl. Instrum. Methods Phys. Res., Sect. A 576, 109 (2007).

[20] B. Henke, E. Gullikson, and J. Davis, At. Data Nucl. Data Tables 54, 181 (1993).

[21] M. Born and E. Wolf, Principles of Optics (Pergamon Press, Oxford, 1980).

[22] M. O. Hasnah, C. Parham, E. D. Pisano, Z. Zhong, O. Oltulu, and D. Chapman, Med. Phys. 32, 549 (2005).

[23] R.D. Rawlings, Materials Science and Engineering (EOLSS Publishers, Oxford, 2009). 